\title{
Relations de pouvoir et égalitarisme sexuel chez un groupe d'étudiants marocains
}

\author{
El Feki Mansour ${ }^{1}$ A. Harakat ${ }^{2}$, J. J. Lévy ${ }^{1}$ J. Otis 1 L. R. Frigault ${ }^{1}$ \\ 1 Département de sexologie, UQAM. Montréal.2 Société marocaine de Sexologie et d'Andrologie
}

\section{RÉSUMÉ}

Afin de vérifier quels sont les différences de sexe qui existent dans le domaine des rapports de pouvoir, de l'égalité dans les rôles sexuels, de même que dans celui du contrôle des pulsions sexuelles parmi les jeunes marocains, 111 étudiants universitaires $(50$ hommes et 61 femmes) ont répondu à un questionnaire auto-administré et anonyme portant sur leur vie sexuelle. Les résultats statistiques indiquent que pour les deux sexes, la sexualité apparaît plus comme un champ de partage que de pouvoir. Ils s'accordent aussi pour considérer que l'initiation des relations sexuelles fait partie des responsabilités masculines, tout comme les initiatives dans l'emploi du préservatif et les modalités de l'expression de la sexualité. Par contre, les hommes considèrent qu'ils sont plus à même d'exprimer des demandes sexuelles que les femmes. Celles-ci sont mieux capables de contrôler leurs pulsions sexuelles et de moduler leur vie sexuelle. Les résultats indiquent aussi la présence d'un double standard dans l'entrée dans la vie sexuelle, les homme étant significativement plus actifs que les femmes. Ces données confirment en grande partie les analyses anthropologiques plus qualitatives réalisées sur la sexualité au Maroc.

Mots-clés : relations de pouvoirs, rôles sexuels, conduites sexuelles.
La question de la sexualité au Maroc a reçu un traitement essentiellement anthropologique comme en témoignent, entre autres, les travaux de Naamane-Guessous [9],Mernissi [7, 8], Rosander [11] et Davis et Davis [1, 2]. Ceux-ci insistent sur les déterminants religieux et socioculturels qui contraignent l'expression de la sexualité. Ils mettent en relief la présence de tensions sexuelles notables entre les hommes et les femmes, en particulier dans le monde rural. Les processus de socialisation différentiels entre les garçons et les filles qui entraînent la mise en place du développement d'identités sexuelles fortement contrastées et de rapports de pouvoir inégalitaires s'expriment aussi dans la sphère sexuelle où l'érotisme masculin tend à être valorisé aux dépens de celui de la femme. Les hommes ont ainsi la possibilité d'explorer le champ sexuel avant le mariage. Celui-ci s'accompagne des privilèges liés à la polygynie, aux relations extra- maritales et aux règles de répudiation. Chez les femmes, les valeurs liées à la honte, la pudeur et la virginité, valeurs encore dominantes chez les jeunes adultes des deux sexes [8] inhibent la réponse sexuelle qui ne peut être exprimée théoriquement que dans le cadre du mariage.

Ainsi l'entrée dans la vie sexuelle est souvent marquée par les rituels de défloration, puis

Correspondance Joseph J. Lévy Département de sexologie Case postale 8888, succursale centre-ville Montréal H3C $3 P 8$ Québec Canada 
continue par la suite d'être dominée par des tensions et des dysfonctions majeures au plan de la satisfaction sexuelle. Dans les rapports de couple, où le mari est à la fois présent et absent, on constate le repli des femmes sur la fonction maternelle, le plus souvent sous la supervision de la belle-mère l'une des figures d'autorité majeures dans le contexte familial.

Ces configurations semblent cependant être actuellement soumises à des transformations liées à la modernisation des valeurs liées à l'influence des messages transmis par les médias contemporains dominés par les modèles occidentaux et arabes ( télévision, musique populaire), les voyages et l'éducation [1]. Les médias en particulier servent à transmettre des modèles nouveaux de conduites et de styles de relations amoureuses, de mariage et de couple dont l'acceptation reste cependant souvent ambivalente.

Comme le montre Davis [1, 2] dans son étude sur les relations de genre en milieu semi-rural, les conduites sexuelles des jeunes marocains tendent à dévier des normes traditionnelles encore acceptées au plan idéologique. La majorité des jeunes adultes, en particulier les filles, continuent de favoriser le choix de leur conjoint par leurs parents, alors qu'un cinquième d'entre eux considèrent cependant qu'ils auront à jouer un rôle dans cette décision. L'importance du sentiment amoureux, influencé par les médias, semble cependant être de plus en plus significatif dans la définition de la relation de couple, un sentiment qui est perçu comme pouvant cependant s'accommoder des exigences normatives qui entourent le choix du conjoint. La sexualité reste encore un champ tabou et occulté en particulier chez les jeunes filles qui refuseront d'en parler directement, abordant plutôt les émotions qui entourent cette sphère, contrairement aux garçons qui sont plutôt prolixes et directs sur les sujets sexuels.

Ces jeunes adultes, tout en considérant que la virginité au mariage continue d'être importante, auront des pratiques sexuelles pré- maritales. Parmi les jeunes filles, ces pratiques comprennent le baiser, le coït ou des pénétra- tions inter- fémorales et anales qui préservent la virginité. Les garçons, par contre, auront des relations sexuelles avec des prostituées ou des jeunes filles non vierges ou bien des relations homo- érotiques comprenant des masturbations réciproques, des pénétrations interfémorales ou anales comme substitut aux relations hétérosexuelles.

Ces données qualitatives indiquent donc la présence d'un double- standard sexuel qui s'accompagne de demandes contradictoires qui créent un climat de non- confiance, rendant difficiles les rapports d'intimité. Ces données rejoignent les données provenant d'études effectuées en milieu urbain [10] qui montrent la présence d'attitudes sexistes des garçons vis-à-vis des femmes qui sont considérées comme des prostituées, des diablesses et des traîtresses, alors que le mariage est appréhendé.

Cet ensemble de recherches suggère que les jeunes adultes des deux sexes sont confrontés à une phase de transition au plan des valeurs et des normes socioculturelles qui dérivent des conflits entre les modèles traditionnels et modernes, influencés en partie par les représentations médiatiques étrangères au milieu marocain.

Afin de contribuer à mieux cerner les configurations socio sexuelles des jeunes marocains, nous nous proposons dans le cadre de cet article de présenter les résultats préliminaires d'une recherche quantitative portant sur les attitudes face à l'usage du pouvoir ou du partage sur le plan sexuel, l'égalitarisme dans les rôles érotiques, la perception de la sexualité et du contrôle des pulsions sexuelles personnelles.

\section{MÉTHODOLOGIE}

\section{Les sujets}

Un questionnaire auto- administré et anonyme a été distribué en 1997 à 111 jeunes adultes marocains fréquentant des institutions universitaires. La population comprenait 50 hommes et 61 femmes. La moyenne d'âge était de 20,5 ans. Il existe peu de variations dans les 
croyances religieuses, $91,9 \%$ se définissant comme beaucoup ou très croyants. Par contre la pratique est plus partagée puisque $51,4 \%$ disaient ne jamais ou rarement pratiquer alors que $48,6 \%$ pratiquaient quelquefois ou souvent. La très grande majorité, $93,7 \%$, était célibataire.

\section{Le questionnaire}

Le questionnaire reprend celui de la recherche provinciale menée sur les risques face au sida, les relations de pouvoir et les styles de communication sexuelle chez les lycéens du Québec. Dans le cadre de cette présentation les dimensions suivantes ont été retenues:

1. les attitudes face aux relations de pouvoir ou d'échange dans la sphère sexuelle.

2. les attitudes face aux rôles dans la sphère sexuelle.

3. la perception de sa propre sexualité, du contrôle des pulsions sexuelles et des conduites à risques. Afin de mesurer ces attitudes un ensemble de 27 énoncés ont été définis et chaque question se situait sur une échelle ordinale allant de 1 (désaccord) à 5 (accord).

\section{1. les attitudes face aux relations de pou- voir ou d'échange dans la sphère sexuelle.}

Afin de mesurer ces attitudes, un ensemble de huit énoncés a été défini. L'analyse factorielle a permis de dégager la présence de deux facteurs qui forment des sous- échelles. La première renvoie à la perception de la sexualité comme l'expression de rapports d'échange et comprend quatre énoncés:

a) la sexualité est une forme de communication entre deux personnes;

b) la sexualité est un échange de faveurs entre deux personnes consentantes;

c) la sexualité est d'abord une façon de prendre son propre plaisir par l'intermédiaire de l'autre;

d) la sexualité c'est d'abord l'acte de se donner l'un à l'autre. L'ensemble de ces questions forme une échelle allant de 4 (sexualité sans échange) à 20 (sexualité comme échange) (alpha de Cronbach, 0,60).
La seconde échelle renvoie à la perception de la sexualité comme l'expression de rapports de pouvoir et comprend également quatre énoncés:

a) une bonne façon d'exciter sexuellement quelqu'un est de lui donner l'impression qu'on le domine;

b) il est acceptable d'utiliser la sexualité pour gagner du pouvoir sur l'autre;

c) dans le domaine sexuel, il est acceptable de mettre de la pression sur le-la partenaire;

d) la sexualité doit s'exprimer sans qu'une personne exerce du pouvoir sur l'autre. L'ensemble de ces questions forme une échelle allant de 4 (sexualité sans pouvoir) à 20 (sexualité avec pouvoir) (alpha de Cronbach, $0,57)$.

\section{2. les attitudes face aux rôles dans la sphère sexuelle.}

Afin de mesurer ces attitudes 9 énoncés ont été définis. L'analyse factorielle a permis de dégager trois facteurs. Le premier porte sur l'égalitarisme dans l'initiation de la relation sexuelle et comprend deux énoncés:

a) c'est davantage aux hommes qu'aux femmes d'amorcer une relation sexuelle;

b) lors d'une relation sexuelle c'est aux hommes plutôt qu'aux femmes de décider du moment pour mettre le condom.

Ces deux questions forment une échelle allant de 2 (égalitarisme) à 10 (inégalitarisme) (alpha de Cronbach, 0,58). La seconde échelle porte sur la responsabilité liée à l'usage du préservatif et comprend aussi deux questions:

a) lors d'une relation sexuelle, les femmes devraient laisser leur partenaire installer le condom plutôt que de le faire elles-mêmes;

b) il est plus acceptable pour les femmes que pour les hommes de proposer de mettre un condom.

Ces questions forme une échelle allant de 2 (égalitarisme dans l'initiation) à 10 (inégalitarisme dans l'initiation) (alpha de Cronbach, $0,48)$. La troisième sous- échelle porte sur l'égalitarisme dans les attitudes face à l'ex- 
pression de la sexualité et comprend 4 énoncés:

a) les femmes doivent se sentir moins libres que les hommes de draguer;

b) il est plus acceptable pour les femmes que pour les hommes de dire non à une relation sexuelle;

c) lors d'une relation sexuelle, l'homme devrait prendre un rôle plus actif que la femme;

d) les hommes sont plus habiles que les femmes en matière sexuelle.

Cet ensemble d'énoncés forme une échelle allant de 4 (rapports inégalitaires dans l'expression de la sexualité) à 20 (rapports égalitaires) (alpha de Cronbach, 0,61).

\section{3. la perception de sa propre sexualité, du contrôle des pulsions sexuelles et des conduites à risques.}

Dix questions ont été formulées. L'analyse factorielle a permis de dégager 3 facteurs:

\section{L'initiative dans les demandes sexuelles} qui comprenait 3 questions:

a) Je serais capable de dire à quelqu'un comment il peut me faire plaisir sexuellement;

b) Je serais capable de prendre l'initiative lors d'une relation sexuelle;

c) Je serais capable de demander à quelqu'un de me procurer les stimulations sexuelles dont j'ai envie.

Cet ensemble d'énoncés forme une échelle allant de 3 (non- initiative) à 15 (initiative) (alpha de Cronbach, 0,79). Le second facteur renvoie au contrôle des pulsions sexuelles et comprend 3 énoncés:

a) Je pourrais contrôler mes pulsions sexuelles;

b) Si j'étais excité(e), j'aurais des relations sexuelles avec n'importe qui;

c) Si j'étais excité(e), je ferais n'importe quoi. Cet ensemble d'énoncés forme une échelle allant de 3 (non contrôle) à 15 (contrôle) (alpha de Cronbach, 0,54).

Le troisième facteur renvoie à la capacité de moduler son activité sexuelle et comprend 4 énoncés: a) Je serais capable de fréquenter quelqu'un sans me sentier obligé(e) d'avoir une relation sexuelle;

b) Je serais capable de choisir quand et avec qui avoir des relations sexuelles;

c) Je serais capable de refuser une pratique sexuelle avec laquelle je ne suis pas à l'aise;

d) Peu importe mon niveau d'excitation sexuelle, je serais capable de dire non à des activités sexuelles à risques.

Cet ensemble d'énoncés forme une échelle allant de 4 (faible modulation) à 20 (forte modulation) (alpha de Cronbach, 0,56). Afin de vérifier les variations sur ces échelles selon le sexe et d'autres variables indépendantes (âge, activité sexuelle, pratique religieuse), le t de Student a été utilisé. Le X2 a été utilisé pour la variable portant sur la vie sexuelle selon le sexe.

\section{RÉSULTATS}

Le tableau 1 montre les résultats significatifs suivants:

\section{1. les attitudes face aux relations de pou- voir ou d'échange dans la sphère sexuel- le.}

On ne constate pas de différences significatives selon le sexe pour la dimension partage ou pouvoir, mais les moyennes indiquent que les répondants des deux sexes sont enclins à désapprouver l'usage du pouvoir dans la sphère sexuelle et à être plus en faveur d'une approche plus synergique.

2. les attitudes face aux rôles dans la sphère sexuelle.

En ce qui concerne l'égalitarisme dans l'initiation de la relation sexuelle et de l'usage du préservatif, les hommes et les femmes ne différent pas mais ils ont tendance à reconnaître une responsabilité plus grande des hommes dans ce domaine.

Sur la responsabilité liée à l'usage du préservatif, les hommes et les femmes ne différent pas mais ils sont enclins à être d'accord pour considérer que c'est à l'homme d'en proposer l'usage. 
Tableau 1 : Variations significatives selon le sexe pour les différentes échelles

\begin{tabular}{l|l|l|l|l} 
Échelles & homme & femmes & $\mathbf{t}$ & $\mathbf{p}$ \\
\hline $\begin{array}{l}\text { Sexualité } \\
\text { comme partage }\end{array}$ & 14.8 & 13.7 & & NS \\
$\begin{array}{l}\text { Sexualité } \\
\text { comme pouvoir }\end{array}$ & 7.7 & 7.6 & NS \\
$\begin{array}{l}\text { Initiation des rela- } \\
\text { tions sexuelles }\end{array}$ & 6.6 & 6.6 & NS \\
$\begin{array}{l}\text { Responsabilité face } \\
\text { au préservatif } \\
\text { Égalitarisme }\end{array}$ & 9.0 & 9.7 & 11.5 & NS \\
$\begin{array}{l}\text { Demandes } \\
\text { sexuelles }\end{array}$ & 12.2 & 9.6 & -4.43 & .001 \\
$\begin{array}{l}\text { Contrôle des } \\
\text { pulsions } \\
\begin{array}{l}\text { Modulation de } \\
\text { la sexualité }\end{array}\end{array}$ & 11.6 & 12.8 & 2.58 & .01 \\
\hline
\end{tabular}

Quant à la prise en charge dans l'expression de la sexualité, il n'y pas de différences entre les hommes et les femmes. Cependant les moyennes montrent que les deux sexes sont d'accord pour considérer que ce sont les hommes qui devraient être le plus en charge de cette dimension.

\section{3. la perception de sa propre sexualité, du contrôle des pulsions sexuelles et des conduites à risques.}

C'est dans ce domaine que les différences entre les hommes et les femmes sont les plus claires. Ainsi, dans l'expression des demandes sexuelles, les hommes sont significativement plus portés à considérer qu'ils sont capables d'exprimer leurs désirs et leurs besoins dans ce domaine alors que les femmes semblent moins à même de faire connaitre leurs desiderata.

Le contrôle des pulsions sexuelles est par contre significativement plus fort chez les femmes que chez les hommes, tout comme la capacité de maîtriser et de moduler leur vie sexuelle. Les autres variables, comme l'âge, la pratique religieuse n'interviennent pas significativement sur ces échelles. Par contre, l'analyse des résultats montre qu'il existe des différences significatives dans l'entrée de la vie sexuelle selon le sexe. Ainsi 92,0\% des hommes rapportent avoir eu des relations sexuelles alors que $90,2 \%$ des femmes n'ont pas eu de telles relations. Compte tenu qu'il existe une corrélation très forte entre le fait d'être de sexe masculin et l'entrée dans la vie sexuelle, l'effet de cette variable sur les différentes échelles a été ignoré.

\section{DISCUSSION}

L'objectif de notre recherche avait pour but de dégager les différences existant entre des hommes et des femmes fréquentant des institutions éducatives marocaines quant aux attitudes face aux rapports de pouvoir ou de partage dans la rencontre sexuelle, à l'égalité dans la sphère sexuelle, érotique et contraceptive, de même qu'en ce qui a trait au contrôle des pulsions sexuelles.

On constate ainsi qu'il n'existe pas de différences de sexe quant aux attitudes face au pouvoir ou de partage dans les relations sexuelles. Les relations de partage tendent cependant à être plus privilégiées. On peut y voir sans doute l'impact du romantisme et des modèles de couple prônés par la télévision et qui favorisent les valeurs d'échange et d'attention [1, 2]. Cependant, même si les rapports d'autorité semblent s'estomper pour faire place à un érotisme plus axé sur la communication et le partage du plaisir, les hommes et les femmes ne renoncent pas à une division des rôles dans la sphère érotique. L'homme reste encore de façon significative le responsable des initiatives, que ce soit dans l'amorce des relations sexuelles, les décisions entourant le port du préservatif ou les rôles dans le déroulement des activités sexuelles.

Ces résultats recoupent les données anthropologiques quant à l'importance de la passivité dans le rapport sexuel. Comme le note Naamane-Guessous ([9] p. 243-244) " [...] seule une sur quatre parmi les femmes mariées ose 
prendre l'initiative de faire l'amour avec son mari.[...] Dans leur ensemble, elles considèrent que l'acte sexuel est une affaire exclusivement masculine, et pour elles ce n'est qu'un devoir[...] Mais certaines d'entre elles refusent de laisser paraître leur désir, même quand il est réel, avant que le mari n'ait fait le premier pas; cette passivité féminine est le résultat de toute une éducation puritaine." Les hommes et les femmes maintiennent donc des attitudes inégalitaires, les premiers assumant un plus grand contrôle de ces aspects inter- personnels.

Certains de ces contrastes se prolongent dans la sphère de la perception de la vie sexuelle personnelle. Ainsi, les femmes disent être plus réticentes à exprimer leurs demandes érotiques, alors que les hommes sont plus à même de faire connaître leurs besoins. Cette différence rejoint là encore les observations de Naamane-Guessous [9] : les sentiments de honte et de pudeur (hchouma) constituent l'un des socles de la sexualité féminine, ce qui empêche la communication et souvent l'obtention du plaisir, entraînant de ce fait des insatisfactions sexuelles notables chez les femmes. Il existe par ailleurs un dimorphisme significatif dans la perception du contrôle des pulsions sexuelles, plus fort chez les femmes que les hommes.

On retrouve ce contraste dans d'autres recherches qui indiquent que la sexualité masculine se construit sur la performance et la puissance sexuelles, alors que celle des femmes semble être basée sur une expression sexuelle plus maîtrisée [5]. De même dans les conceptions islamiques " [...] la femme musulmane est contrainte pour sa part de se conformer au modèle de vertu imposé par le contexte culturel; ce modèle lui enjoint en particulier d'étouffer ses pulsions sexuelles et de maîtriser en elle toute fonction érotique" ([9], p. 236), alors que les hommes sont au contraire poussés à explorer le champ des rapports érotiques avec moins de contraintes.

Les femmes se disent aussi mieux capables de maîtriser et de moduler leur vie sexuelle, de même que de choisir le registre des activités sexuelles qu'elles sont prêtes à accepter. Ce résultat semble rejoindre les données qualitatives de Namane- Guessous : les contraintes liées aux stratégies matrimoniales et aux fréquentations obligent les femmes à circonscrire les pratiques sexuelles qu'elles se permettront afin de garder leur valeur matrimoniale. Cette problématique des rapports de genre parmi les jeunes marocains n'est pas non plus sans rejoindre, à certains égards, les données de recherches transculturelles qui montrent le maintien d'une inégalité marquée dans la sphère érotique $[3,4,5,6]$, avec pour corollaire, des difficultés du contrôle des pulsions sexuelles axées essentiellement sur la pénétration vaginale. Les schémas sociaux liées à la sexualité féminine orientent par contre son expression vers un érotisme où la passivité, le renoncement au plaisir et le devoir sexuel seraient plus prégnants. Souvent, les femmes ne pourraient exprimer librement leurs besoins sexuels, à moins d'accepter que leur sexualité ne soit perçue comme déviante ou fondée sur des rapports de promiscuité. Leur manque d'assurance, leurs difficultés à communiquer au plan sexuel, leur perception des hommes comme acteurs sexuels plus compétents, interviendraient ainsi pour amplifier l'expression d'une sexualité sous l'emprise du pouvoir masculin. Dans ces rapports de pouvoir basés sur le genre, les hommes contrôleraient donc le contexte de l'interaction érotique, son contenu et son déroulement [12].

Notons par ailleurs les écarts énormes qui existent dans les conduites sexuelles des hommes et des femmes puisque ces dernières sont bien moins enclines à avoir des relations sexuelles que leur collégues masculins. S'agitil là de l'expression d'un contrôle socio- sexuel particulièrement accentué envers les femmes ou bien de l'effet des codes d'expression sexuelle rattachés à la pudeur chez ces dernières? Il est difficile en l'absence de données complémentaires de répondre clairement à ces questions. Néanmoins, ces résultats suggèrent le maintien d'un double standard sexuel qui marque les limites de la modernisation de la sexualité chez les jeunes marocains. 


\section{CONCLUSIONS}

Cette étude portait sur les attitudes face au pouvoir dans la sphère sexuelle, l'égalité dans les rôles sexuels et le contrôle des pulsions sexuelles parmi des étudiants d'origine marocaine. Les résultats montrent, comme le suggérant les études anthropologiques sur la sexualité marocaine, que par certains aspects les attitudes à l'égard des dimensions sexuelles varient selon les dimensions considérées.

Ainsi, si les attitudes s'éloignent des modèles où domine le recours au pouvoir au profit des valeurs de partage et d'échange, l'expression érotique reste encore marquée par la prédominance de l'homme dans la modulation du déroulement de la vie sexuelle, ce qui rejoint le paradigme culturel érotique marocain de base et qui se prolonge dans une conception marquée par le dimorphisme de genre dans l'expression des besoins sexuels, de la maîtrise des pulsions et de la modulation de la vie érotique.

Les femmes sont ainsi soumises à un plus grand contrôle social qui se manifeste par une gestion plus répressive de leur vie sexuelle, confirmée par le fait que la virginité-un symbole majeur de l'idéologie socio -sexuelle marocaine- reste encore très prégnante parmi les femmes par obligation ou par choix , alors qu'au contraire les hommes se trouvent déjà engagés dans une carrière sexuelle.

Ces lignes de clivage suggérent que les caractéristiques des relations familiales et sexuelles continuent d'être dominées par des conceptions et des conduites qui contribueront aux problèmes socio- psychologiques et sexologiques que les chercheurs maghrébins se sont attachés à décrire. Une intervention sexologique au plan d'une éducation sexuelle poussée pourrait aider à mieux circonscrire ces aspects et en réduire les effets dans les groupes d'âge préoccupés à développer une vie sexuelle plus épanouie.

\section{RÉFÉRENCES}

1. DAVIS, D.A. , AND DAVIS, S.S. .Sexual values in a Moroccan Town. In W.J. Lonner and R.S. Malpass (Eds.)Psychology and culture. Needham Heights: Allyn and Bacon 1993, pp. 225-230.
2. DAVIS, S.S., \& DAVIS, D.A. Adolescence in a Moroccan town: Making social sense. New Brunswick: Rutgers University Press 1989.

3. HOLLAND, J., RAMAZANOGLU, C., SCOTT, S.,THOMSON, R.. «Desire, Risk and Control: The Body as a Site ofContestation». In L. Doyal, J. Naidoo, T. Wilton (eds.) AIDS:Setting a $\mathrm{F}$ e $\mathrm{m}$ i $\mathrm{n}$ i s t Agenda, London: Taylor \& Francis Ltd, 1994a, 61-79.

4. HOLLAND, J., RAMAZANOGLU, C., SHARPE, S.,THOMSON, R. «Achieving Masculine Sexuality: Young Men's Strategiesfor Managing Vulnerability». In L. Doyal, J. Naidoo, T. Wilton(eds.) AIDS: Setting a Feminist Agenda, London: Taylor \& Francis Ltd., 1994b, 122-148.

5. HOLLAND, J., ROMAZANOGLU, C., SCOTT, S.,SHARPER, S. ET THOMSON, R. Sex gender and power: young women'ssexuality in the shadow of Aids. Sociology of Health et Illness, 1990, 12, 336-350.

6. HOLLAND, J., ROMAZANOGLU, C., SCOTT, S.,SHARPER, S. ET THOMSON, R . Risk, power and the possibility ofpleasure: young women and safer sex. Aids Care, 1992, 4,273-283.

7. MERNISSI, F. Behind theveil: Male-female Relations in a Modern Muslim Society. Cambridge: Schenkman 1975.

8. MERNISSI, F. Sexe,idéologie, islam. Paris: Tierce 1983.

9. NAAMANE-GUESSOUS, S. . Au-dela de toute pudeur: La sexualite feminine au Maroc. Paris, Casablanca: Éditions Khartala-EDDIF, 1991.

10. OUZZI, A.. ThePsychology of Adolescence: A field study of the psycho-sociologicalattitudes of the Moroccan adolescent. Publié par manshuratmajallat ad-dirasat an-nafsiya wa-terbawiya, B.P.823, Rabat . 1986.

11. ROSANDER, E.E.. Womenin borderland. Managing muslim identity where Morocco meets Spain. Stockholm Studies in Social anthropology , 1991.

12. SPRECHER, S ET MCKINNEY, K. Sexuality. Newbury Park, Calif. : Sage. 6372, (1993.

\section{ABSTRACT}

Power relations and equality in sexual roles in a group of moroccans students

El Feki Mansour, A. HaraKaf, J.J.Lévy et al

In order to verify what are the differences in the field of power relations, equality in the sphere of sexual roles as well as the control of sexual drives among young Moroccans, 111 university students(50 men and 61 women) 
have answered an anonymous self-administred questionnaire dealing with their sexual lives. Statistical results show that for both sexes, sexuality appears more as a sharing experience than a power one. Both sexes consider also that it is the prerogative of men to initiate sexual relations as well to decide the use of condom. Men consider that they are more able to express their sexual demands than women. The latter are more in control of their sexual drives and of the modulation of their sexual life. The results indicate also the presence of a sexual double standard with men being more sexually active than women. These data confirm on the whole the qualitative data provided by anthropological research on sexuality in Morocco.

Key-words : power relations, sexual roles, sexual drives 